\title{
Experience with the use of a sheet-shaped body vibrometer in infants admitted to the neonatal intensive care unit
}

\author{
Manabu KEMMOCHI ${ }^{1 *}$, Takamasa KOGURE ${ }^{2}$, Masanori KANEKO $^{1}$, Shuji ISHIDA ${ }^{1}$, Ayano Yamaguchi', Mari OOKA ${ }^{1}$, Yuichiro \\ YOKOZEKI ${ }^{1}$ and Hideaki SENZAKI ${ }^{1}$
}

${ }^{1}$ Kitasato University School of Medicine, Department of Pediatrics, Japan

${ }^{2}$ Paramount Bed Sleep Research Laboratory, PARAMOUNT BED CO., LTD, Japan

\section{Introduction}

For preterm infants and neonates who are at risk of apnea with high heart rate variability, vital function monitoring is essential in the neonatal intensive care unit (NICU). When preterm infants whose skin layers are fragile are placed on an electrocardiographic monitor, its electrode pads cause skin injury [1]. It is also a cause of both mental and physical stress for infants. Importantly, stress during the time when the brain is rapidly developing is known to affect subsequent neurobehavioral development [2]. Therefore, development of monitors for minimizing stress is awaited. In this context, sheet-shaped monitors are considered ideal. In newborn nurseries where full-term infants are usually managed, a sheet-shaped apnea alarm, which is placed under a mattress, is often used. Apnea occurring in full-term infants, which is often secondary apnea caused by infection, hypothermia, etc., can occur even in neonates without any underlying disease [3,4]. Thus, the alarm is routinely set. However, this sheet-shaped apnea alarm aims to detect apnea depending on the presence or absence of thoracic motion but cannot measure respiratory rate or heart rate. Meanwhile, because the sheet-shaped alarm monitor does not impose any stress on neonates and is less expensive than electrocardiographic monitors, it is widely distributed $[5,6]$. No validation study has been conducted on the use of a sheet-shaped monitor for measuring heart rate and respiratory rate in NICU neonates, and such monitors are, in fact, not widespread at NICU setting. In this study, a sheet-shaped body vibrometer (SBV) used primarily for adults, which allows sleep pattern, heart rate, and respiratory rate to be checked without being attached to the subjects, was employed in NICU neonates/infants, in order to examine whether SBV is feasible in terms of safety. When it cannot be used, we investigated what points should be improved. We report herein the results.

\section{Material and methods}

Nemuri SCAN $^{\bullet}$ (NN-1110, Paramount Bed Co., Ltd., Tokyo, Japan) was used as an SBV in this study. The SBV is placed under a mattress for use. Studies conducted on adults have shown that SBV can measure heart rate and respiratory rate [7] determine whether a subject is asleep, awake, or away from the bed [8] and perform rough estimation of itch severity [9] based on body motion; and detect moderate or severe obstructive sleep apnea syndrome based on changes in respiratory movement [10]. The SBV was placed on beds of NICU neonates/infants for 24 hours. First, the presence or absence of adverse events was assessed to determine the safety. Anticipated adverse events were bedsore, frequent vomiting, changes in body temperature by \pm 2 ${ }^{\circ} \mathrm{C}$ or more, and removal of tubes and other devices during transport.
Moreover, to confirm the accuracy of data from SBV, heart rate and respiratory rate measured with SBV were compared with those obtained from an electrocardiographic monitor (MX800 or 700, Philips) that was attached to all NICU infants. An SBV for monitoring was placed on the neonatal bed by folding it in half (Figure 1).

This study was conducted with an approval of the ethics committee of Kitasato University School of Medicine (B-1552). We explained the objectives of this study to family members of all subjects and obtained their written consent. Of infants admitted to our NICU during the period between September 2015 and January 2016, 39 infants whose parents or guardian gave consent to participation in this study were included. First, safety was assessed in these 39 patients. Then, the calculation rate and accuracy of SBV monitoring were assessed by dividing the infants into three groups $(<2000 \mathrm{~g}, 2000-2500 \mathrm{~g}$, and $>2500 \mathrm{~g}$ ) according to body weight at measurement.

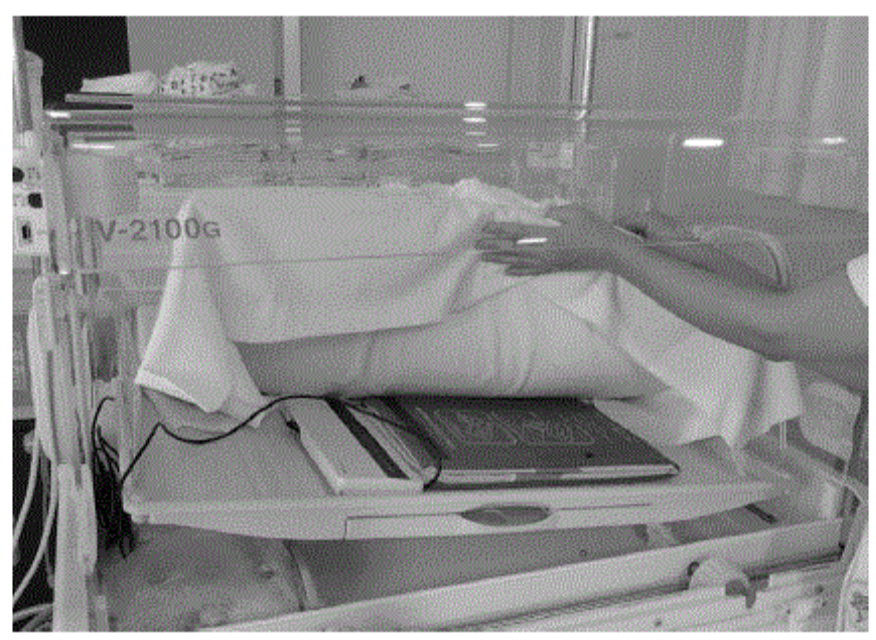

Figure 1. Setting of the sheet-shaped body vibrometer

${ }^{*}$ Correspondence to: Manabu KEMMOCHI, Kitasato University School of Medicine, Department of Pediatrics, 1-15-1 Kitasato Minami-ku Sagamihara Kanagawa, Japan, Tel. +81-42-778-8111, E-mail: kenmochi@med.kitasato-u.ac.jp

Key words: ampullary region, flat intraepithelial neoplasia, polypoid lesion, tubule-villous adenoma, Vater's papilla

Received: December 18, 2018; Accepted: January 03, 2019; Published: January 07,2019 


\section{Calculation method}

SBV automatically calculates both respiratory rate and heart rate once every 5 seconds. When body motion or a signal-to-noise (SN) ratio was automatically shown to be large with SBV, neither respiratory rate nor heart rate was calculated. Thus, we calculated a ratio of the time for which calculation of heart rate and respiratory rate with SBV was achieved to the entire monitoring time (calculation rate).

Furthermore, the respiratory rate and heart rate, as calculated with SBV, were compared with those obtained from an electrocardiographic monitor to assess the accuracy of SBV monitoring. Regarding accuracy assessment, because the rates were recorded every minute by the electrocardiographic monitor and calculated differently by these devices, the timing of calculating the rates could not be completely synchronized between SBV and the electrocardiographic monitor. Thus, when respiratory rate and heart rate calculated with SBV deviated by less than $\pm 10 \%$ from those obtained from the electrocardiographic monitor, these rates were considered to be in agreement. The agreement in accuracy was assessed on a 3-point scale: good agreement (GA) defined as an agreement rate of $80 \%$ or higher, moderate agreement (MA) defined as $40 \%$ to $80 \%$, and poor agreement (PA) defined as $40 \%$ or lower.

\section{Results}

Background characteristics of 39 monitored infants are shown in Table 1. These infants included 10 extremely-low-birth-weight (ELBW) infants, 3 infants with congenital heart disease (CHD), and 5 infants diagnosed with chronic lung disease (CLD) at monitoring. In all infants, a 24-hour monitoring was possible without inducing any adverse events.

Next, accuracy of SBV monitoring was assessed by excluding intubated infants in whom the involvement of external vibration markedly reduced both calculation rate and accuracy. Finally, both the calculation rate and accuracy were assessed in 31 infants, excluding 8 infants comprising 1 with insufficient electrocardiographic data and 7 intubated infants (high-frequency oscillation [HFO] mode in 1 and intermittent mandatory ventilation [IMV] mode in 6). Background characteristics of these 31 infants are shown in Table 2. These infants included 7 ELBW infants, 3 with CHD, and 5 with CLD.

Both calculation rate and accuracy increased in association with an increase in body weight at monitoring (Figures 2 and 3). That is, both parameter levels were poor in infants weighing $<2000 \mathrm{~g}$ and relatively good in infants weighing $>2500$ g. Figure 4 shows examples of actual measurements. In one infant with GA measurements and another infant with PA measurements (Figure 4-(1) and (2)), most segments without plotted SBV data correspond to moments with frequent body motion. In intubated infants, artifacts presumably derived from the ventilator were so great that the accuracy was markedly reduced (Figure 4-(3)).

\section{Discussion}

The currently used sheet-shaped apnea alarms aim to detect thoracic movement and sound alarm when the thorax stops moving for 10-20 seconds. These devices are incapable of monitoring respiration or heart beats. However, monitoring of vital function, such as respiration and heart beats, is essential at NICU settings. At present, monitoring of respiratory rate and heart rate requires the use of an electrocardiographic monitor or pulse oximeter (arterial oxyhemoglobin saturation). To use these devices, electrode pads and other components must be attached to infants, and attachment of the components imposes stress on them.
Table 1. Background characteristics of All 39 infants

\begin{tabular}{|l|c|}
\hline $\mathbf{n}$ & $\mathbf{3 9}$ \\
\hline Gestational week (wk) & 33.0 \\
\hline Birth weight (g) & 2051 \\
\hline Body weight at monitoring (g) & 2091 \\
\hline Age at monitoring (day) & 19.2 \\
\hline Boys & 25 \\
\hline Endotracheal intubation & 7 \\
\hline Mechanical ventilation & 15 \\
\hline Ventilation mode & 1 \\
\hline HFO & 6 \\
\hline IMV & 8 \\
\hline NPPV & 6 \\
\hline Oxygen administration alone & \\
\hline Bed & 15 \\
\hline Incubator & 14 \\
\hline Infant & 10 \\
\hline Cot & \\
\hline
\end{tabular}

Table 2. Background characteristics of 31 infants included in the assessment of calculation rate and accuracy

\begin{tabular}{|l|c|}
\hline $\mathbf{n}$ & $\mathbf{3 1}$ \\
\hline Gestational week (wk) & 33.1 \\
\hline Birth weight $(\mathrm{g})$ & 2019 \\
\hline Body weight at monitoring $(\mathrm{g})$ & 2067 \\
\hline Age at monitoring (day) & 21.4 \\
\hline Boys & 19 \\
\hline NPPV & 8 \\
\hline Oxygen administration & 6 \\
\hline Bed & \\
\hline incubator & 12 \\
\hline Infant & 10 \\
\hline Cot & 9 \\
\hline
\end{tabular}

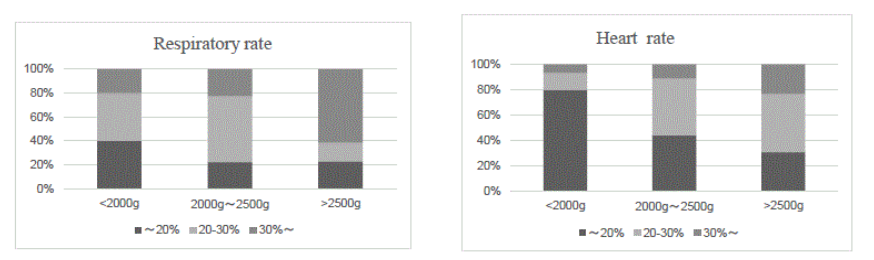

Figure 2. Comparison of calculation rates according to body weight

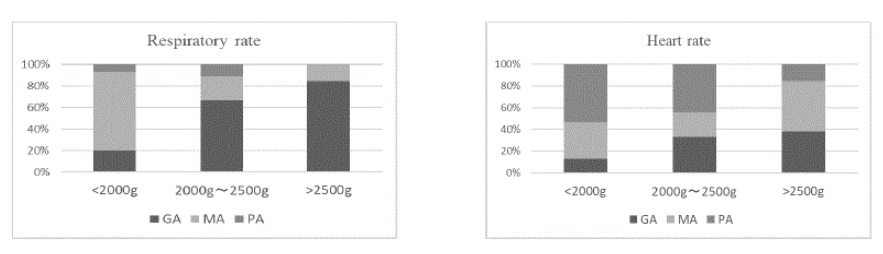

Figure 3. Comparison of accuracy according to body weight. GA: agreement rate of $80 \%$ or higher, MA: $40 \%$ to $80 \%$, PA: $40 \%$ or lower

An SBV is only placed under a mat to monitor infants, and no stress is thus imposed. Actually, it is widely used for adults at care settings; SBV is useful in providing care at night to monitor fluctuations in heart rate and respiratory rate and whether patients are asleep or awake. ${ }^{7}$ This study aimed to determine whether SBV could be used for neonates/infants whose body motion is small. Additionally, it served as a preliminary study to explore necessary modifications when SBV could not be used.

The results of this study revealed the safety of SBV without inducing any adverse events. SBV could measure heart rate and 
1)

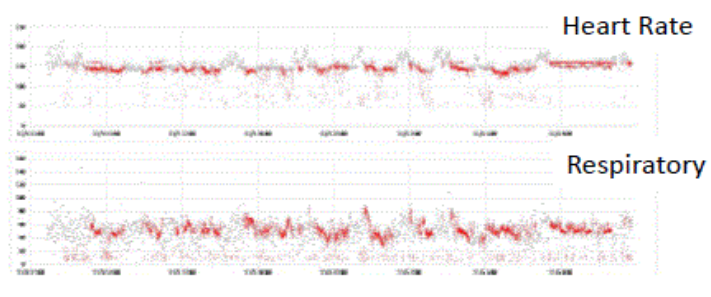

2)

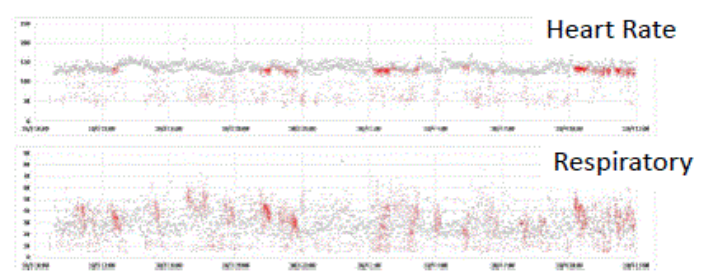

3)

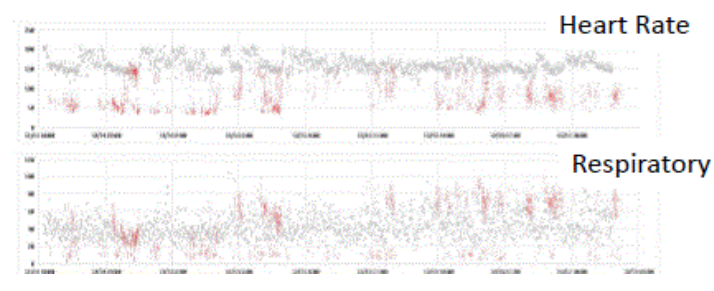

Figure 4. Monitoring results

Gray dots: measurements from the heart rate monitor. Red dots: measurements from the sheet-shaped body vibrometer

(1) A case of good agreement (GA)

(2) A case of poor agreement (PA)

(3) An intubated infant

respiratory rate at certain accuracy for infants with a relatively large body weight of $2500 \mathrm{~g}$ or more, though the calculation rate needs to be improved. However, the present SBV was incapable of monitoring both heart rate and respiratory rate in infants weighing less than $2500 \mathrm{~g}$; thus, it might be employed as an apnea alarm, but requires improvement in hardware and software for monitoring respiratory rate and heart rate. Both calculation rate and accuracy of SBV monitoring were reduced in infants, including low-birth-weight infants. For infants with severe periodic breathing and those with frequent apneic episodes, when heart rate interval and heart beat intensity greatly fluctuated due to respiratory rate fluctuation, the $\mathrm{SN}$ ratio was determined to be low. As a result, both calculation rate and accuracy of SBV monitoring tended to be reduced. Furthermore, in infants placed on mechanical ventilator with endotracheal intubation, heart-beat signals could not be differentiated from high-frequency artifacts caused by operation of the ventilator; therefore, the heart rate was found particularly difficult to measure.

The present study has two main limitations. First, because adultsized SBV was used by folding it in half, it was not suited to the size of the cot, infant, and couveuse. Second, despite great differences in respiratory rate and heart rate between adults and neonates, the algorithm designed for adults was used, without any modification, in the present infants.

In conclusions, monitoring of vital function is essential in preterm and low-birth-weight infants whose vital signs greatly fluctuate. Thus, monitors for minimizing stress and providing reliable data are preferable. SBV has the potential to satisfy these needs. The data from this study may provide insights into the practical use of SBV in the field of neonatology through further hardware and algorithm improvements.

\section{Conflicts of interest}

The authors declare no conflict of interest.

\section{Sources of financial assistance}

This study is partly funded by a research grant from PARAMOUNT BED CO., LTD., and the sheet-shaped body vibrometer (SBV: Nemuri SCAN $^{\circ}$ ) used in this study was provided by PARAMOUNT BED CO., LTD.

\section{References}

1. Wille J, Braamus R, Van Haren WH, van der Werken C (2000) Pulse oximeter-induced digital injury: Frequency rate and possible causative factors. Crit Care Med 28: 3555 3557. [Crossref]

2. Cong X, Wu J, Vittner D, Xu W, Hussain N, et al. (2017) The impact of cumulative pain/stress on neurobehavioral development of preterm infants in the NICU. Early Hum Dev 108: 9-16. [Crossref]

3. Hoppenbrouwers T, Hodgeman JE, Harper RM, et al. (1977) Polygraphic studies of normal infants during the first six months of life: Incidence of apnea and periodic breathing. Pediatrics 60: 418-425. [Crossref]

4. Lee D, Caces R, Kwiatkowski K, Cates D, Rigatto H (1987) A developmental study on types and frequency distribution of short apneas ( 3 to 15 seconds) in term and preterm infants. Pediatr Res 22: 344-349. [Crossref]

5. MacFadyen UM, Borthwick G, Simpson H, McKay M, Neilson J (1988) Monitoring for central apnoea in infancy--limitations of single channel recordings. Arch Dis Child 63: 282-287. [Crossref]

6. Behrens O, Goeschen K, Schneider J (1988) Home-monitoring in children at risk for sudden infant death syndrome. A comparative study of 5 apnea mattresses without cables. Fortschr Med 106: 645-650. [Crossref]

7. Kogure T (2017) Sleep characteristics and improvement in elderly care. Journal of care and behavioral sciences for the elderly 22: 17-27.

8. Kogure T, Shirakawa S, Shimokawa M, Hosokawa Y (2011) Automatic sleep/wake scoring from body motion in bed: validation of a newly developed sensor placed under a mattress. J Physiol Anthropol 30: 103-109. [Crossref]

9. Kogure T, Ebata T (2018) Activity during sleep measured by a sheet-shaped body vibrometer and the severity of atopic dermatitis in adults: A comparison with wrist actigraphy. J Clin Sleep Med 14: 199-204. [Crossref]

10. Kogure T, Kobayashi M, Okawa T, et al. (2017) Validation of a sheet-shaped body vibrometer for screening of obstructive sleep apnea. Drug Discov Ther 11: 126-132. [Crossref]

Copyright: (C2019 KEMMOCHI M. This is an open-access article distributed under the terms of the Creative Commons Attribution License, which permits unrestricted use, distribution, and reproduction in any medium, provided the original author and source are credited. 